\title{
Clinical and ethical implications of genetic counselling in familial adenomatous polyposis
}

\author{
A. Fernández-Suárez, C. Cordero Fernández¹, R. García Lozano, A. Pizarro¹, M. Garzón ${ }^{1}$ and A. Núñez \\ Roldán
}

Department of Immunology and 'Digestive Diseases. Virgen del Rocío University Hospitals. Seville, Spain

\begin{abstract}
The association of specific genetic disturbances with the development of hereditary cancer helps us to understand the risk of suffering from it, the possibility of an earlier diagnosis, and the treatment and prevention of this disease. Familial adenomatous polyposis (FAP) is a pre-neoplastic syndrome characterized by the presence of hundreds of adenomatous polyps in the colon, which develop into a carcinoma. FAP can be diagnosed using sequencing techniques to detect mutations in the germinal line of the APC (adenomatous polyposis coli) gene.

The genetic diagnostic approach in families with FAP, previously followed up in the Gastrointestinal Clinic, has both advantages and disadvantages, and places us nearer the disease and patient. Disclosing the results of this genetic test entails relevant problems in clinical practice, which affect the health field and raise legal and ethical issues, along with the familial, occupational, and social implications that knowing the genetic status can have on the patient.

Genetic analysis is rare in normal clinical practice, which involves errors in the interpretation of the results obtained, and during the process of genetic counselling. Specialized multidisciplinary units are necessary for the management of patients with FAP undergoing analysis and appropriate genetic counselling, thus providing an individualized service. The creation of FAP registers and protocols for this healthcare process should optimize the management of these patients and their families.
\end{abstract}

Key words: Familial adenomatous polyposis. APC gene. Molecular diagnostic techniques. Genetic counselling.

Fernández-Suárez A, Cordero Fernández C, García Lozano R, Pizarro A, Garzón M, Núñez Roldán A. Clinical and ethical implications of genetic counselling in familial adenomatous polyposis. Rev Esp Enferm Dig 2005; 97: 654-665.

Recibido: 03-01-05.

Aceptado: 04-01-05.

Correspondencia: Carmen Cordero Fernández. Servicio de Aparato Digestivo. Hospital General. Hospitales Universitarios Virgen del Rocío. Avda. de Manuel Siurot, s/n. 41013 Sevilla. e-mail: carmenc@inicia.com

\section{INTRODUCTION}

The discovery that cancer is the result of mutations in particular genes that interfere in the growth, differentiation and death of cells, and the possibility of finding these mutations, has led to a revolution in the field of the diagnosis, treatment and prevention of this disease. This revolution is especially relevant to cases of familial hereditary cancer.

Colorectal cancer is the second largest cause of cancerrelated death in Spain, after lung cancer. Its development is sporadic in $75 \%$ of patients, while the remaining $25 \%$ are related to a family history of this disease. Familial adenomatous polyposis (FAP) is, with an incidence of 1:8000 newborns, the second most common genetic syndrome predisposing to cancer after hereditary cancer of the colon, a condition not related to polyposis, and is responsible for between 1 and $2 \%$ of all cases of colorectal cancer.

\section{FAMILIAL ADENOMATOUS POLYPOSIS}

FAP is characterized by the presence of hundreds of adenomatous polyps in the colon, which inevitably develop into carcinomas in the absence of early diagnosis and patient treatment using prophylactic colectomy (1). Polyps can develop at very early ages, although this process usually occurs in the second or third decade of life, with a mean age of 16 years. Patients can remain asymptomatic for years but when symptoms develop the risk of suffering from cancer is around $75 \%$. Mean age at cancer onset is 39 years, and the age of death for patients not preventively treated is 42 years. FAP is also characterized by the development of adenomatous polyps with the capacity of becoming malignant in sections of the upper digestive tract, especially the duodenum. For polyps located in the papilla, this malignant transformation occurs in $12 \%$ of patients; therefore, even after treatment, 
the degeneration of these polyps is a cause of death for many of these patients.

There is an attenuated variant of FAP (AFAP), which is characterized by a lower number of polyps in the colon (generally less than 50), with a predominance in the right colon, later development, and degeneration 10 to 15 years later than in classic FAP. Upper sections of the gastrointestinal tract are affected with numerous hyperplastic polyps in the gastric fundus, as well as with duodenal adenomas (2).

In both polyposis (in the classic as well as the attenuated variant), along with the risk of developing colorectal or duodenal cancer, patients have an added risk of suffering from thyroid cancer, hepatoblastoma, desmoid tumors (3), tumors of the biliary tract, and other phenotypic disturbances such as congenital hypertrophy of retinal pigment epithelium (CHEPR) (3).

FAP is an autosomal dominant inherited disorder with a penetration of $100 \%$; therefore, every child born from an affected patient presumably has a 50\% chance of suffering from this disease (4). It is essential, therefore, that detailed genealogical trees of families affected are performed, and that a systematic control of patients and their direct relatives is achieved, with the objective of carrying out an early diagnosis of the disease and thus avoiding the development of colorectal cancer. When genetic tests are not available, direct family members should be examined by colonoscopy at 14-16 years of age, and by subsequent sigmoidoscopy every year until the age of 40-45 years, when the disease can be reasonably excluded. Gastroduodenal endoscopy must be carried out to investigate potential involvement of the upper sections. In the case of family members with non-carrier status, investigations should begin at least at 15 years of age. One colonoscopy and 29 sigmoidoscopies would be required to clarify this fact.

\section{GENETICS OF FAP}

Numerous studies have been carried out to ascertain the origin of this disease. Thus, in 1987, using genetic mapping techniques, a gene was located in the long arm of chromosome 5 (5). Later, in 1991, the gene responsible for the disease was identified (6) and designated adenomatous polyposis coli (APC). This gene is made up of 8535 base pairs and 21 exons, and codes for a protein of 2843 amino acids. Exon 15 contains $75 \%$ of the coding sequence and is also the place where mutations affecting the gene are most commonly found. It is a tumor-suppressing gene (7), since its alteration occurs in the initial stages of the process transforming a normal tissue into a neoplastic one.

The APC protein plays a fundamental role in the regulation of intra-cellular $\beta$-catenin levels, decreasing its concentration in the cytoplasm and stabilizing tissues (1). This regulation is carried out by a degradation of $\beta$-catenin, thus inhibiting the Wnt signaling pathway towards the interior of the cell. The accumulation of $\beta$-catenin causes a loss of cell adhesion, the transcription of homeotic genes involved in the polarity and architecture of tissues, and an activation of cell proliferation. Therefore, mutations in the APC gene give rise to an altered and non-functional protein, which results in uncontrolled growth of epithelial cells, thus giving rise to polyps. The hypothesis described by Kundson $(1,7)$ to explain the natural history of FAP at a genetic level postulates that, in addition to an initial mutation in the germinal line, a second event must occur to bring about malignancy. This second event could be an additional somatic mutation in one of the polyps or a loss of heterozygosity. The type of germinal mutation in the APC gene appears to determine the type of the second event. When it occurs between codons 1194 and 1392, then an allelic loss is selected; on the other hand, when located outside this area, it favors somatic truncating mutations.

In the majority of patients with FAP, mutations in the germinal line of the APC gene have been found. Ninetyfive per cent of these mutations are non-directional, and give rise to a truncated protein. One third of germinal line mutations occur in the 1061 and 1309 codons. The rest are homogenously distributed between codons 200 and 1600 , no mutation having been described outside these margins. The APC gene is also implicated in the AFAP phenotype, although mutations found in the germinal line are different from the FAP phenotype (2).

Several phenotype/genotype associations have been described, and there is considerable variation in the expression of specific phenotypes within families and in isolated individuals with the same mutation. In this sense, mutations between codons 1250-1464 are associated with the presence of a large number of colorectal polyps (> 5000) with a severe phenotype (8). Similarly, the mutation in codon 1309 gives rise to early manifestations of the disease and a serious phenotype. Mutations occurring in the 5' (codons 78-163) or 3' ends of the gene, or in the post-transcriptional processing region of exon 9 , give rise to AFAP (9). Mutations between codons 457-144 are associated with CHEPR (3). Extracolonic manifestations appear to occur more often in patients with mutations between codons 1445 and 1578, according to some authors, or between codons 1395 and 1493, according to other authors $(3,10)$. A different evolution at the mucosa of the rectal stump has been described, and the risk of cancer at this level in patients undergoing prophylactic subtotal colectomy depends on the location of the APC gene mutation. For this reason a genetic analysis could also determine the appropriate surgical treatment for each patient (11). However, correlations between genotype and phenotype need to be further clarified to allow for the potential design of genetic probes in the future, which will exclusively tackle the study of key locations in this gene in order to gain insight into the evolution and prognosis of individual patients. 


\section{GENETIC DIAGNOSIS}

The benefit of carrying out genetic tests in this disease is endorsed by the American Society of Clinical Oncology (ASCO), which in 1997, and in accordance with the indication of molecular tests and the benefit obtained in knowing the results of such tests (12), included FAP in group 1. This group contains those families with hereditary cancer syndromes where a positive or negative result involves changes in medical action or prenatal management, and for whom a genetic test is considered a part of the normal management of affected families.

FAP can be diagnosed genetically by detecting mutations in the germinal line of the APC gene (13-15). To meet this goal, DNA is obtained from circulating leucocytes in peripheral blood. Later, using sequencing techniques, the mutation within the gene can be seen in the patient affected by FAP. Once the mutation is located in indicated patients (those patients diagnosed with FAP using clinical criteria) the rest of the family members may be promptly diagnosed with almost $100 \%$ certainty, provided that the whole family has the same mutation. This study can discern which family members have inherited the mutated gene and are going to develop the disease (which can also affect their offspring), and which have not inherited this mutation, with neither they nor their offspring ever requiring subsequent follow up $(13,14,16)$.

\section{GENETIC COUNSELLING}

The function of a genetic counselling clinic is the evaluation of the risk present in a particular family meeting the criteria of hereditary cancer where one of their members may suffer from this particular type of cancer. Since we are in a position to offer a genetic test that may evaluate that risk, we must discuss with the patient the possibilities available in the event of positive, negative or inconclusive results, as well as the implications of all these situations.

Disclosing the results of a genetic test often entails important problems in clinical practice, many of which remain to be solved. These problems have health, legal and ethical repercussions, and are not easily understood by patients and often by the doctors caring for them (14). It is not uncommon for patients not to understand the voluntary nature and privacy of these studies, or their significance, as well as the familial, occupational and social implications that knowing one's genetic status may have. In many cases, it is difficult to know which is the best approach in those patients who have a positive genetic test and -we believe- cannot deal with this information; or in those, who we believe can potentially use this information against other family members. Answers to these problems are a part of genetic counselling.
Lastly, it should be mentioned that on numerous occasions genetic tests carried out in health centers with the isolated efforts and initiative of individuals show significant gaps in standardization and the regulation of the genetic counselling process.

\section{PHASES OF GENETIC COUNSELLING}

\section{Preparation and decision-making phase}

Before obtaining a blood sample, the patient must sign an informed consent form with specific information on the test to be performed, as well as on the consequences derived from the type of result obtained; the possibility that the test might be negative, positive or inconclusive; the possibility of transmission to offspring; the technical reliability of the test; the risks of emotional distress; and lastly, the familial, occupational and social implications.

The autonomy principle recognizes that individuals have the capacity to deliberate and then decide. This principle plays a relevant part in situations related to studies of genetic predisposition, since an individual who is correctly informed and has the capacity to make decisions and accept their consequences can decide whether or not to undergo and learn the results of a genetic study. At times, due to family pressures or other reasons, genetic studies are carried out on individuals at risk who subsequently decide that they do not want to know its result. According to ethical principles, an individual does not have to be informed about the result regardless of whether he/she is a carrier or not, since the autonomy principle takes preference over welfare.

The welfare principle obliges doctors to always act in the best interest of the patient or individual. On occasion several ethical principles can come into conflict, such as, for example, the individual benefit regarding the welfare of third parties and the right to autonomy regarding that of welfare, as mentioned earlier. In this sense American bioethics tends to value the principle of autonomy above welfare, while in our study the principle of welfare is set before that of autonomy. According to the U.S. National Institute of Health and the recommendations of the European Council, an individual's consent must be obtained before revealing that individual's personal information to third parties. However, this confidentiality can be ignored under certain circumstances, for example where not revealing such information may endanger third parties. Studies of genetic predisposition have medical, psychological and social repercussions for the individual as well as for the family. The best way to prevent the conflicts and harm that may arise from these types of studies is to offer prior genetic advice and discuss potential consequences. 


\section{Waiting phase}

During this period the patient experiences great uncertainty, which appears to increase with the waiting period as anxiety can be seen to rise (17). For this reason, and knowing that sequencing techniques involve a lot of work and require a relatively long time to be carried out (especially with a gene as extensive as APC), the health personnel responsible for these procedures should be informed of the consequences of unnecessary or unjustified delays.

\section{Communication phase of the results and follow-up}

Communication of results should be a process voluntarily accepted by persons undergoing the test. The subjects under study are responsible for calling in order to find out whether the results are available and to arrange a new appointment to discuss them. This is because in certain cases people having undergone the test reassess their situation during this waiting period, and may come to decide that they do not want to know the results. Also, there can be others who in their particular situation may want to know the results at a later date, when their circumstances improve.

As regards the way of communicating the results, it is desirable to have a personal meeting either with each member of the family on an individual basis or with all of them together. Each of these options has advantages and disadvantages. Information on the results of a particular person is more private and allows the subject to expresses all his/her doubts and worries when given separately. On the other hand, when informing the whole family as a group, such information is homogeneous even if questions are more difficult to pose, which prevents interpretation errors and misunderstandings regarding the meaning.

After communicating a positive result, the various studies to be carried out and the necessary treatment should be discussed (a subject that should have been raised at the first consultation). At this time it is important to offer the patient psychiatric help, when necessary. Also, at the same time, this interview should provide insight into the patient's psychological and emotional status.

Psychological problems which may arise from knowing the results of a genetic test are, by nature, very variable, whether the result is positive or negative. A very good summary of the potential consequences of learning one's genetic status is provided by Petersen et al. (18). In view of a positive test result, we find positive consequences such as decreased uncertainty and the possibility of making decisions regarding subsequent health care, as well as those involving different aspects of life: work, economy or reproduction. On the other hand, negative components include the fact that subjects lose their hope of escaping from the disease, and become angry with their circumstances or their affected relatives. They may feel different or stigmatized by everybody else, may suffer from depression or either transient or chronic anxiety, lose self-confidence, and feel guilty regarding their offspring or even jealous regarding their siblings with a negative test. If the result is negative, then positive feelings include relief, loss of guilt regarding their offspring, and greater self confidence. A negative aspect is a potential "survivor guilt" feeling.

\section{CHILDREN AND ADOLESCENTS}

As has been shown, we have here an oncological syndrome which affects children and very young people in which genetic information is crucial to develop a series of preventive measures, which in some cases may save the child's life. The principle of autonomy laid out in the General Law of Health (Ley General de Sanidad), as was already commented on earlier, considers the possibility of refusing pertinent genetic tests on behalf of the interested party, and even the right "not to know", which is also considered in our rules, laws and regulations. In this case, using ethical criteria, the potential clash between individual rights as derived from the principle of autonomy and the rights of third parties (children or parents at risk) to health must be considered.

In these cases it is essential that the whole family be involved in decision making (19). To achieve this objective, parents and children (depending on their age) alike must jointly be informed and educated, and a parental consent as well as the assent of the child or adolescent must be obtained. Discussing the possible advantages and disadvantages will help them reach a consensus solution. Also topics such as the concealment or holding back of information must be treated as a priority, thus avoiding undesirable situations within the family.

The child must be involved in the preparation consultation so that he/she may clearly understand the purpose and implications of the test (18). Parents generally tend to underestimate as well as overestimate the knowledge that their children have of FAP. In any case, we must not offer excessive information to a young person, which could cause increased anxiety; we should try to come to an agreement with his/her parents on the contents before the consultation with the child. Parents should know the results of genetic tests a few minutes before their children, with the aim of having an opportunity to control their emotions and avoid alarming the child unnecessarily. In a survey carried out on adult patients with FAP (20), 93\% said that they would have liked their children to have been offered the genetic test at birth or during infancy, indicating that an age between 10 and 12 years was ideal to start introducing the concept of FAP. In our experience, in the majority of cases parents do not want their children to have the genetic test until diagnostic or therapeutic measures are needed. 
Psychological problems that may arise in children in the short term as well as in the long term have been analyzed in various studies $(21,22)$. On evaluating children at risk of suffering from FAP three months after receiving the results of their genetic tests, no clinically significant psychological symptoms were found either in them or their parents. However, sub-clinical changes developed in mutation-positive children, and major indices of anxiety were present in cases where the mother was affected by FAP. When the effects were evaluated at 3, 12 and 23 to 55 months later, no clinically significant changes in the scores of psychological tests were found in these children or their parents. However, like in short-term studies, children with a positive mutation who also had a positive sibling presented with significant sub-clinical increases in depressive symptoms. Also, some children with negative results but a positive sibling had increased anxiety symptoms. Anyway, these same authors pointed out the necessity of studies with a larger number of families and a more complete composition of individuals as well as positive and negative cases.

\section{EXPERIENCE IN OUR HOSPITAL}

In our Department of Digestive Diseases, 20 families affected by FAP have been studied ( 87 members, 34 persons clinically diagnosed with FAP, and 53 at risk for this disease, without knowledge of their genetic status). Patients with FAP were diagnosed by means of total colonoscopy. In all families a genetic analysis of the APC gene was carried out by the Immunology Department. The procedure consisted of a direct sequencing of the first 15 exons in the APC gene following amplification by means of PCR on DNA obtained from a sample of peripheral blood (23); an ABI PRISM 310 Genetic Analyzer (Applied Biosystem) automatic sequencer was used. The sequences obtained were compared -using the Navigator software- to the sequences and mutations for FAP in existing data bases. Once the causal mutation is identified in the index patient, an analysis of the affected exon is carried out in the remaining family members.

The mutation responsible for the disease was detected in sixteen of twenty families studied, since certain mutations which affect the APC gene may not be detected with the present strategy (for example, mutations in the gene promoter). However, mutations in the APC gene were detected in all patients previously diagnosed with FAP (except in the four families where no mutation had been found yet). Of the 87 individuals examined, a mutation was found in 37, of which 26 had already been clinically diagnosed. Eleven patients had been diagnosed with FAP using the genetic test before the development of clinical symptoms. On the other hand, 26 individuals at risk for this disease (between 4 and 40 years old) were discharged, as they were free of the causal mutation.
All mutations found (except two affecting the splicing process) caused the synthesis of truncated proteins due to the presence of a stop codon in their mRNA. The majority of mutations were located in exon 15 (8-16), with the rest being distributed in different exons $(14,11,5,6$ and $8)$. The two mutations affecting the post-transcriptional process were located in the margins of introns 6 and 7 . The most frequent type of mutation consisted of small deletions ( 8 cases), followed by changes of a base ( 5 cases) and small insertions ( 2 cases).

The data obtained confirmed a wide heterogeneity in the location of mutations associated with FAP throughout the APC gene, which results in a wide variation in the clinical expression of the disease. However, and despite the diversity of mutations found, the Spanish population with FAP has not been investigated at a genetic level in a great number of cases, with the exception of some works published in the literature $(15,23)$. Also, a subsequent analysis of the clinical variables and histopathology for each mutation found would be necessary in order to establish correlations between genotype and phenotype, which would help understand the possible outcome and prognosis of the disease.

\section{CLINICAL REPERCUSSIONS AND GENETIC COUNSELLING}

The use of this genetic test eliminates the need for annual follow-up with colonoscopies and sigmoidoscopies in all patients and subjects at risk where a family mutation is not found. On the other hand, the detection of this mutation in eleven individuals that had not yet developed the disease, means that they may be followed up in a more meticulous way, as well as adopting the necessary prophylactic measures. Finally, it has substantially improved the prevention of colorectal cancer by getting patients who did not want to undergo regular colonoscopies to accept the molecular analysis, also encouraging the remaining family members who were reluctant to be studied.

But without a doubt, the crucial and most important aspect is that, due to this genetic counselling based on molecular studies, non-carriers of the disease will be assured that they will not suffer from this disease and will not transmit it in any way to their future offspring, with the resulting psychological relief and the elimination of numerous medical consultations and examinations, along with their corresponding health and social costs (24). In this way, according to the data shown in the present study, this genetic test eliminates the need for annual monitoring (based on invasive tests) regarding 26 patients between 4 and 40 years of age. For an approximate estimation of the saving in health costs, if we assume an average age of 20 years for these 26 patients, 1,950 unnecessary medical consultations and 650 colonoscopies would have been avoided. 
Against this, and although it is beyond doubt that the identification of affected subjects is a benefit for them as regards adopting prophylactic measures, evidence exists of negative psychological reactions derived from the knowledge of a positive result. This is even more significant when we consider the fact that the disease affects to a great extent children and young people (in the group of patients studied, out of eleven asymptomatic individuals, six were younger than 20 years and nine younger than 30 years of age), who will have to make important decisions regarding all aspects of their familial, occupational and social life.

\section{CONCLUSIONS}

Genetic analyses are scarcely used in normal clinical practice, and on occasion health professionals do not interpret their results correctly, or proceed correctly during the genetic counselling process (14). These facts support the benefit of having specialized units available for the management of FAP, in which genetic analyses can be carried out and appropriate genetic counselling is available. The creation of FAP registries and the establishment of protocols for this health process should optimize the management of these patients and their families $(25,26)$.

The wide heterogeneity and complexity of the challenges we have leads us to treat the genetic information obtained with extreme caution. For this reason, genetic counselling should be carried out in multidisciplinary units in hospital centers, as well as in all health units where genetic tests are carried out and communicated to patients. This would represent individualized, specialized care approaching each case in a unique way, thus ensuring maximum efficiency for the health process in all its fields. Also, these units would accumulate experience and add valuable knowledge to the field of genetic counselling, both in general and according to regional social and cultural peculiarities, which would strengthen even more a quality health service.

\section{REFERENCES}

1. Fearnhead NS, Britton MP, Bodmer WF. The ABC of APC. Hum Mol Genet 2001; 10(7): 721-33

2. Lynch HT, Smyrk T, McGinn T, Lanspa S, Cavalieri J, Lynch J, et al. Attenuated Familial Adenomatous Polyposis. Cancer 1995; 76 (12): 2427-33.

3. Caspari R, Olschwang S, Friedl W. Familial adenomatous polyposis: desmoid tumors and lack of ophthalmic lesions (CHRPE) associated with APC mutations beyond codon 1444. Hum Mol Genet 1995; 4: 337-40.

4. Burt RW, Groden J. The genetic and molecular diagnosis of adenomatous polyposis coli (editorial). Gastroenterology 1993; 104: 1211-4.

5. Bodmer W, Bailey C, Bodmer J, Bussey H, Ellis A, Gorman P, et al. Localization of the gene for familial adenomatosus polyposis on chromosome 5. Nature 1987; 328: 614-6.

6. Groden J, Thliveris A, Samowitz W, Carlson M, Gelbert L, Albertsen
$\mathrm{H}$, et al. Identification and characterization of the familial adenomatous polyposis coli gene. Cell 1991; 66: 589-600.

7. Goss KH, Groden J. Biology of the adenomatous polyposis coli tumor suppressor. J Clin Oncol 2000; 18 (9): 1967-79.

8. Spirio LN, Samowitz W, Robertson J. Alelles of APC modulate the frequency and classes of mutation that lead to colon polyps. Nature Genet 1998; 20: 385-8.

9. Brensinger JD, Laken SJ, Luce MC. Variable phenotype of familial adenomatous polyposis in pedigrees with $3^{\prime}$ mutation in the APC gene. Gut 1998; 43: 548-52.

10. Olschwang S, Tiret A, Laurent-Puig P. Restriction of ocular fundus lesions to a specific subgroup of APC mutations in adenomatous polyposis coli patients. Cell 1993; 75: 959-68.

11. Vasen HFA, van der Luijt RB, Slors JFM, Buskens E, de Ruiter P, Baeten CGM, et al. Molecular genetic tests as a guide to surgical management of familial adenomatous polyposis. Lancet 1996; 348: 433-5.

12. Garber JE, Offit K, Olopade OI, Fink D, Barbasch A, Barr P, et al. The American Society of Clinical Oncology position on genetic testing: implications for health care providers: workshop no. 4. Cancer 1997; 80 (Supl. 3): 632-4.

13. Powell SM, Petersen GM, Drush AJ, Booker S, Jen J, Giardiello FM, et al. Molecular diagnosis of familial adenomatous polyposis. N Engl J Med 1993; 329: 1982-7.

14. Giardiello FM, Brensinger JD, Petersen GM, Luce MC, Hylind LM, Bacon JA, et al. The use and interpretation of commercial APC gene testing for familial adenomatous polyposis. N Eng J Med 1997; 336 : 823-7.

15. Ruiz-Ponte C, Vega A, Carracedo A, Barros F. Mutation analysis of the adenomatous polyposis coli (APC) gene in northwest Spanish patients with familial adenomatous polyposis (FAP) and sporadic colorectal cancer. Hum Mutat 2001; 18: 355.

16. Peterson GM, Bresinger JD, Johnson KA, Giardiello FM. Genetic testing and counselling for hereditary forms of colorectal cancer. American Cancer Society 1999; 86: 2540-50.

17. Broadstock M, Michie S, Gray J, Mackay J, Marteau TM. The psychological consequences of offering mutation searching in the family for those at risk of hereditary breast and ovarian cancer--a pilot study. Psychooncology 2000; 9 (6): 537-48.

18. Petersen GM, Boyd PA. Gene tests and counselling for colorectal cancer risk: lessons from familial polyposis. J Natl Cancer Inst Monogr 1995; 17: 67-71.

19. American Society of Human Genetics Board of Directors, American College of Medical Genetics Board of Directors. Points to consider: ethical, legal, and psychosocial implications of genetic testing in children and adolescents. Am J Hum Genet 1995; 57 (5): 1233-41.

20. Whitelaw S, Northover JM, Hodgson SV. Attitudes to predictive DNA testing in familial adenomatous polyposis. J Med Genet 1996; 33 (7): 540-3.

21. Codori AM, Petersen GM, Boyd PA, Brandt J, Giardiello FM. Genetic testing for cancer in children. Short-term psychological effect. Arch Pediatr Adolesc Med 1996; 150 (11): 1131-8.

22. Codori AM, Zawacki KL, Petersen GM, Miglioretti DL, Bacon JA, Trimbath JD, et al. Genetic testing for hereditary colorectal cancer in children: long-term psychological effects. Am J Med Genet 2003; 116A (2): $117-28$

23. García-Lozano JR, Cordero C, Fernández-Suárez A, Encarnación M, Pizarro A, Núñez-Roldán A. APC germline mutations in Southern Spanish patients with familial adenomatous polyposis: Genotypephenotype correlations and identification of 8 novel mutations. Genet Test 2005; 9 (1): 37-40.

24. Bapat B, Noorani H, Cohen Z, Berk T, Mitri A, Gallie B, et al. Cost comparison of predictive genetic testing versus conventional clinical screening for familial adenomatous polyposis. Gut 1999; 44 (5): 698703.

25. Dunlop MG. Guidance on gastrointestinal surveillance for hereditary non-polyposis colorectal cancer, familial adenomatous polyposis, juvenile polyposis, and Peutz-Jeghers syndrome. Gut 2002; 51: V21V27.

26. Bulow S. Results of national registration of familial adenomatous polyposis. Gut 2003; 52: 742-6. 